\title{
Medication Reimbursement Model and Cost Savings in a Canadian Ambulatory Hemodialysis Program
}

\author{
Jo-Anne S Wilson, Jaclyn Tran, Annette Veith, David Landry, Heather Neville, Cindy Kelly, \\ Steven Soroka, and Kenneth West
}

\section{INTRODUCTION}

$\mathrm{H}$ emodialysis (HD) units in Canada provide dialysis treatment and medication therapy to patients with advanced chronic kidney disease. IV iron is commonly indicated for iron deficiency anemia in patients with chronic kidney disease, and is often administered to patients while they receive dialysis in an outpatient clinic. ${ }^{1}$ Each provincial government allocates funding to its renal program to operate outpatient HD clinics. The provincial renal program is responsible for its own staffing and drug budgets but has experienced an increase in demand to treat more patients requiring $\mathrm{HD} .{ }^{2}$ Drugs administered in outpatient clinics are not defined as medically necessary services within the Canada Health Act, instead, patients use a variety of sources to fully or partially cover prescription drug costs, which may include private or public insurance plans. ${ }^{3,4}$ In the authors' province, public drug coverage is offered through the Nova Scotia Pharmacare Programs, which are available for seniors, families with no or limited private drug insurance, and residents receiving community services. ${ }^{5}$ IV iron is a benefit within the Pharmacare drug programs, provided the prescriber completes an exception status form indicating that the patient requires IV iron for management of anemia associated with chronic kidney disease.

In 2013, our hospital developed and approved a policy for drugs used in ambulatory care to help address resource issues and provide consistent and equitable funding of drugs and drug administration in outpatient clinics. ${ }^{6}$ A policy working group, whose members consisted of a pharmacist, a social worker, a lawyer, a bioethicist, and senior managers, clarified the organization's ethical and legal principles and values regarding drug funding. The policy provided a consistent approach to promote fairness and equity, while reducing drug costs to address sustainability issues within the health care system. The hospital became the payer of last resort, which ensured that patients who could not afford to pay for their medications would still be able to receive the needed medications. Accessing patients' existing public or private insurance was for cost-recovery purposes only (not revenue generation) and was not to unduly affect the time to treatment. ${ }^{6}$ Medications that fell under the policy were drugs that might be administered in an outpatient setting but were not required to be given in hospital (e.g., administration in private infusion clinics), ${ }^{7}$ excluding specific medications required to perform procedures or treatments, medications included in the province's high-cost drug program, and insured systemic therapies for cancer. ${ }^{6}$

There are limited data in Canada on approaches to changing drug reimbursement for medications administered in outpatient settings from a hospital-funded model to public or private insurance. Our organization published a pilot study that tested and further informed the hospital's policy by exploring drug coverage options for outpatient therapy with rituximab in 39 patients with rheumatoid arthritis. ${ }^{7}$ The pilot study showed that $87 \%$ of patients had public or private insurance, and making use of that insurance resulted in savings of $\$ 304700$ for the ambulatory care program. Most patients reported that they felt supported by the hospital throughout the pilot and were confident in having their doses administered at a private infusion clinic. However, concerns about the infusion facility were identified, and clinically significant delays occurred, which were attributable to the insurance coverage process. These unintended effects were addressed to mitigate harms and maintain a patient-centred approach. ${ }^{7}$ In Saskatchewan, the Saskatoon Cancer Centre explored sharing the costs of supportive cancer medications with private insurance to restrict public insurance coverage to patients who had no insurance. ${ }^{8}$ Pharmacy students were employed to interview patients waiting for chemotherapy; these interviews showed that $40 \%$ of the patients had private drug insurance that could be utilized for supportive cancer medications. ${ }^{8}$ 
We sought to implement our hospital's payer-of-last-resort drug policy for ambulatory care and to evaluate a reimbursement model for IV iron in several dialysis units in our region.

\section{DESCRIPTION OF PROGRAM}

The Nova Scotia Central Zone's renal program provides HD to about 400 patients per year. The program consists of 3 hospital in-centre HD units (300 patients) and 4 satellite HD units (100 patients). Over the past 2 years, the number of patients requiring $\mathrm{HD}$ in this region has increased by nearly $7 \%$ per year (unpublished data). To manage the increasing costs of delivering dialysis medications, a new reimbursement model was developed, applying the principles of the institution's policy for funding medications used in ambulatory care.

IV iron was the target medication selected for this initiative because it is one of the medications most commonly prescribed and administered during dialysis. In addition, IV iron is a benefit under the publicly funded provincial Pharmacare Programs. Our renal program uses a nurse- and pharmacist-led anemia management protocol for IV iron and erythropoietin-stimulating agents, which is based on standards of care for anemia in patients with chronic kidney disease. ${ }^{9} 10$ Engagement and support from renal program managers, pharmacists, nursing staff, physicians, and social workers were obtained for this initiative. Starting in March 2015, a 6-month feasibility pilot was conducted at one of the smaller in-centre HD units (50 patients) to identify and resolve any perceived or actual barriers before full implementation throughout the renal program. Standardized education was provided to all renal program staff to ensure that patients would receive a consistent message regarding the change in coverage for dialysis medication. All dialysis patients received a letter outlining how medications used in the renal program are funded. Drug coverage information was collected from each patient, drug plan coverage forms were completed as required, and prescriptions for IV iron were faxed to a single community pharmacy (designated through the hospital procurement process). The community pharmacy billed the patient's public or private insurance, and any copayments or deductibles remaining were billed to the renal program. A patient-specific supply of IV iron was delivered by the community pharmacy to the dialysis unit for storage and administration by staff. Feedback from patients and staff was key in shaping the medication reimbursement model.

In September 2015, two larger in-centre HD units began implementing this medication initiative; however, without dedicated staff to interview patients and conduct follow-up with the community pharmacy, implementation was successful in only $60 \%$ of patients. It was realized that it would be helpful to have a dedicated resource person, with knowledge of drug access navigation, to lead the dialysis medication reimbursement program across all dialysis units, to ensure consistent medication refills and ongoing patient enrollment, and thus to realize the potential cost savings. A funding proposal for a pharmacy practice assistant (PPA) to serve as the drug access navigator was submitted and approved by senior management. The analysis supporting this proposal showed that the cost of the PPA could potentially be offset by efficient and effective application of the existing ambulatory drug funding policy to dialysis medications; the PPA would also be available to support other core areas of the renal program (i.e., conducting medication reconciliation, managing inventory, preparing medications, and navigating coverage for other medications).

In April 2017, the renal program hired a PPA (A.V.) to support drug access navigation and to lead implementation of the funding program for ambulatory dialysis medication for about 400 patients receiving hemodialysis in the $7 \mathrm{HD}$ units. The PPA worked closely with all stakeholders involved in the project to adapt procedures to meet the specific needs of each dialysis unit. Figure 1 outlines the steps in the ambulatory dialysis medication reimbursement model. The PPA oversees the management and reimbursement components of the funding model for the $7 \mathrm{HD}$ units. For the 2 larger in-centre dialysis units, the PPA obtains new or refill prescriptions for IV iron, completes any necessary drug coverage forms with prescribers, receives delivery of the IV iron from the community pharmacy daily (or as needed), and organizes the patient-specific supply in the various dialysis units. The PPA maintains inventory records using an index card system. A card is affixed to each patient's own supply of IV iron; when the balance declines to 2 vials, nursing staff place the card in the PPA's bin, which prompts the PPA to obtain a refill from the community pharmacy. In addition, the PPA receives and reviews a copy of the prescription receipts, which detail the amount billed to private or public insurance as well as the copayments or deductibles that are billed to the renal program. The community pharmacy maintains a database of the billing information, and reports are generated monthly and reviewed with the team.

To expedite collection of drug coverage information and the patient's supply of IV iron before the patient starts dialysis, nurses in the outpatient nephrology clinic give to patients the letter explaining how medications in the renal program are funded; the nurses also obtain information about private or public drug insurance from the patient. If a patient requires IV iron and the prescription has not yet been obtained from the community pharmacy, each dialysis unit has a small ward stock supply $(5-10$ vials) of IV iron that can be "borrowed" until the patient's own prescription is available. In the satellite dialysis units and one smaller in-centre HD unit, the PPA's role is to support the charge nurse, who is responsible for obtaining new or refill IV iron prescriptions, completing drug coverage forms with the prescribers, and receiving and organizing the weekly delivery of the patient-specific IV iron from the community pharmacy.

\section{EVALUATION OF PROGRAM}

From April 2017 to March 2018, data were collected from the community pharmacy and the databases maintained by the 


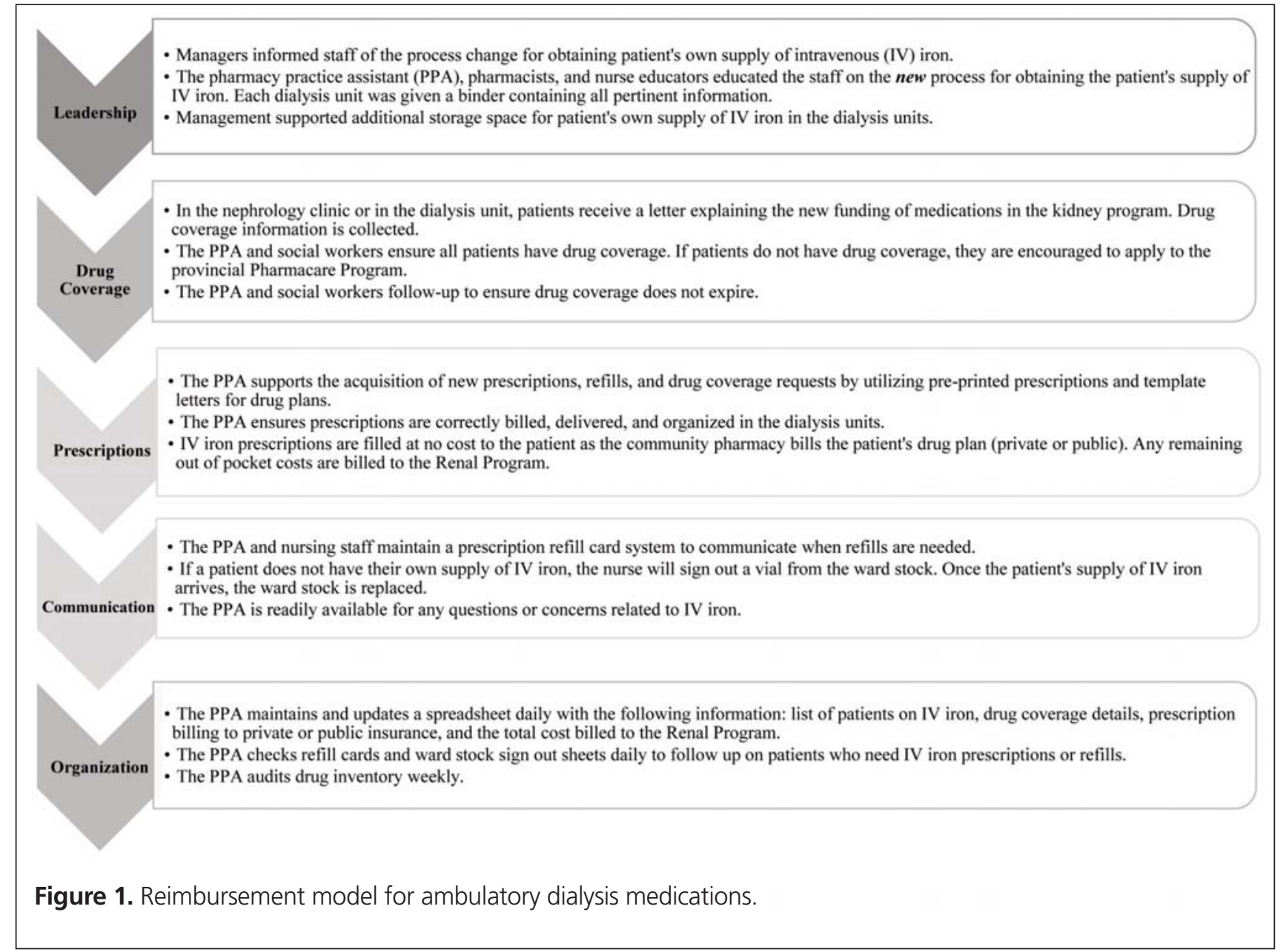

PPA to determine the number of patients who needed IV iron, the number of vials of IV iron dispensed, the type of insurance coverage (private or public) available for each patient's medication, the total costs billed and covered by private and public drug insurance, and the total cost billed to the renal program (including copayments and deductibles for patients with insurance coverage). For patients with no drug insurance, we negotiated with the community pharmacy to pay the same price for IV iron as the hospital was paying for iron sucrose. Because implementation of the program occurred earlier in the in-centre HD units than in the satellite HD units, we used cost data from April 2017 to March 2018 for the in-centre units and from July 2017 to March 2018 for the satellite units. We also determined that the cost of a PPA maintaining the hospital ward stock system for the 7 HD units (including ordering, receiving, storing, and managing IV iron) was equivalent to the cost of having a PPA lead the payer-of-last-resort model for reimbursement for dialysis medication.

A total of 408 patients were enrolled in the ambulatory HD medication reimbursement program: 330 patients in the in-centre units and 78 patients in the satellite units (Table 1). Of these, 253
(62\%) were men and 155 (38\%) were women. A total of 396 patients (97\%) had medication insurance, and $12(3 \%)$ had no medication insurance. Of those with medication insurance, 260 had public insurance and 136 had private medication coverage (Table 1).

Table 2 outlines the total costs billed to and covered by drug insurance, as well as the total cost billed to the renal program for patient copayments, deductibles, and those with no drug insurance coverage (\$90 204.79). If the renal program had paid for all vials dispensed during the evaluation period, the hypothetical ward stock drug cost would have been $\$ 360562.50$. The overall cost savings to the renal program could thus be calculated as $\$ 270357.71$. However, the renal program is itself a provincially funded program, and $64 \%$ of the patients enrolled in the HD medication reimbursement program had public drug insurance. Therefore, we determined that the net cost to the provincial system was $\$ 236689.13$ (including all costs billed to the Pharmacare Programs and the cost of the renal program). The resulting overall cost saving to the provincial system was $\$ 123$ 873.37. These costs and savings are summarized in Table 3 . 
Table 1. Baseline Drug Coverage for Patients Receiving Hemodialysis

Setting for Hemodialysis; No. (\%) of Patients

\begin{tabular}{lcccccc} 
Type of Insurance & \multicolumn{2}{c}{$\begin{array}{c}\text { Satellite Units* } \\
(\boldsymbol{n}=\mathbf{7 8})\end{array}$} & \multicolumn{2}{c}{$\begin{array}{c}\text { In-Centre Unitst } \\
(\boldsymbol{n}=\mathbf{3 3 0 )}\end{array}$} & \multicolumn{2}{c}{$\begin{array}{c}\text { Total } \\
(\boldsymbol{n}=\mathbf{4 0 8})\end{array}$} \\
\hline Public drug insurance & 54 & $(69)$ & 206 & $(62)$ & 260 & $(64)$ \\
Private drug insurance & 17 & $(22)$ & 119 & $(36)$ & 136 & $(33)$ \\
No drug insurance & 7 & $(9)$ & 5 & $(2)$ & 12 & $(3)$ \\
\hline
\end{tabular}

*From July 2017 to March 2018.

†From April 2017 to March 2018

Table 2. Costs Associated with the Hemodialysis Medication Reimbursement Model

\begin{tabular}{lcccc}
$\begin{array}{l}\text { Patient Group, by Type } \\
\text { of Insurance }\end{array}$ & $\begin{array}{c}\text { No. of Vials } \\
\text { Dispensed }\end{array}$ & $\begin{array}{c}\text { Amount Billed to } \\
\text { Insurance (\$) }\end{array}$ & $\begin{array}{c}\text { Cost Covered by } \\
\text { Insurance (\$) }\end{array}$ & $\begin{array}{c}\text { Cost to Renal } \\
\text { Program (\$)* }\end{array}$ \\
\hline Patients with public drug insurance & 4270 & 185484.21 & 146484.34 & 38999.87 \\
Patients with private drug insurance & 4775 & 211224.88 & 181394.96 & 29829.92 \\
Patients with no drug insurance & 570 & NA & NA & 21375.00 \\
Total & 9615 & 396709.09 & 327879.30 & 90204.79 \\
\hline
\end{tabular}

NA = not applicable.

*For patients with insurance, the cost to the renal program was calculated as the total billed to insurance minus the cost covered by insurance. For patients with no insurance, the full cost was covered by the renal program.

\section{Table 3. Renal Program versus Provincial System Costs with the Hemodialysis Medication Reimbursement Model}

\begin{tabular}{|c|c|}
\hline Variable & Cost (\$) \\
\hline $\begin{array}{l}\text { Hypothetical cost for renal program to } \\
\text { supply IV iron as ward stock* }\end{array}$ & 360562.50 \\
\hline Actual cost to renal program† & 90204.79 \\
\hline Savings to renal program‡ & 270357.71 \\
\hline Actual cost to provincial system§ & 236689.13 \\
\hline Savings to provincial system & 123873.37 \\
\hline \multicolumn{2}{|c|}{$\begin{array}{l}\text { *Calculated as total number of vials } \times \text { hospital's cost/vial. } \\
\text { †Total cost billed to the renal program (see Table } 2 \text { ). } \\
\text { \#Calculated as the hypothetical cost minus the actual cost to the } \\
\text { renal program. } \\
\text { §Calculated as the sum of the cost covered by public drug insurance } \\
\text { plus the total cost to the renal program (see Table } 2 \text { ). } \\
\text { १Calculated as the hypothetical cost minus the actual cost to the } \\
\text { provincial system. }\end{array}$} \\
\hline
\end{tabular}

\section{IMPLICATIONS AND SIGNIFICANCE FOR PRACTICE}

With the ever-growing number of patients requiring dialysis, and the need for programs to manage drug expenditures, application of the hospital's policy for funding drugs used in ambulatory care shifts and aligns HD medication coverage with patients' insurance providers. The policy was developed to provide an equitable, consistent process that hospital staff and physicians could use in determining how drugs and their administration are to be funded in outpatient settings. According to this policy, the renal program was designated as the payer of last resort, balancing the need for financial sustainability with societal responsibility to provide options for patients who cannot afford to pay for their own medications. The payer of last resort is defined as the last payer once all other sources of payment, such as patient assistance programs and private and/or public insurance have been billed. ${ }^{6}$ Patients not eligible for coverage by private insurance were encouraged to enroll in the provincial Family Pharmacare Program, which has income-dependent deductibles. ${ }^{5}$ Based on patients' deductibles, the renal program determines whether or not it is in the program's best financial interest to assist with the deductible or to pay the cost of the medication each month. For publicly funded plans, the cost saving is greatest in the months leading up to each patient's yearly renewal month (i.e., April), because patients must satisfy the plan deductible or premium, and a certain percentage $(20 \%-30 \%)$ of each prescription cost is applied until the maximum copayment is reached. Once both the deductible and the maximum copayment have been reached, there is no charge for additional prescriptions. A significant benefit to patients with this reimbursement program is that the renal program pays down the deductibles and copayments associated with IV iron prescriptions. This reduces patients' out-of-pocket costs for the year and allows them to receive other prescriptions that they otherwise might not be able to afford.

Several limitations are associated with this type of reimbursement model. First, for patients with no drug insurance, we were able to negotiate with the community pharmacy a cost for IV iron that was the same as the hospital's cost for iron sucrose. As a result, for those patients without insurance, prescription costs would have been higher if IV iron had been supplied using the community pharmacy's standard pricing. In addition, changes frequently occur with medication coverage for dialysis patients, and overall cost savings therefore tend to decrease or increase proportionally to the number of patients with private insurance. Although staff input informed implementation of the program, staff members' and patients' satisfaction with the process was not evaluated. Furthermore, patients' clinical status was not assessed; however, 
This single copy is for your personal, non-commercial use only.

For permission to reprint multiple copies or to order presentation-ready copies for distribution, contact CJHP at publications@cshp.ca

there were no changes to the anemia management protocol or delays in access to IV iron during the implementation, so it is unlikely that any patients were adversely affected by the change in reimbursement of IV iron. Additionally, for larger HD units without a PPA, it would be important to have a dedicated person leading this type of program to realize the cost savings. We are currently expanding this reimbursement model to include other dialysis medications. Hiring a PPA to lead the HD medication reimbursement model allowed our renal program to maximize cost savings during initial implementation. Since then, however, there has been a shift in responsibilities, with the charge nurse in most HD units now managing the program, with support from the PPA, which in turn allows the PPA to focus on medication reconciliation, inventory control and record keeping, special authorization for high-cost medications, and preparation of medications.

\section{CONCLUSION}

The authors' renal team successfully developed, implemented, and evaluated an innovative and sustainable reimbursement model for a common medication used in ambulatory dialysis. The HD medication reimbursement model promotes cost savings for both the provincially funded renal program and the public drug program, which ultimately contributes to a more sustainable health care system.

\section{References}

1. Shepshelovich D, Rozen-Zvi B, Avni T, Gafter U, Gafter-Gvili A. Intravenous versus oral iron supplementation for the treatment of anemia in CKD: an updated systematic review and meta-analysis. Am J Kidney Dis. 2016;68(5):677-90.

2. Collier R. Number of patients on dialysis increases 30\%. CMAJ. 2017; 189(7):E292.

3. Prescription drug insurance coverage. Ottawa $(\mathrm{ON})$ : Government of Canada; [cited 2018 Oct 25]. Available from: https:/www.canada.ca/en/healthcanada/services/health-care-system/pharmaceuticals/access-insurancecoverage-prescription-medicines.htm

4. Grootendorst P. Beneficiary cost sharing under Canadian provincial prescription drug benefit programs: history and assessment. Can J Clin Pharmacol. 2002;9(2):79-99.

5. Nova Scotia Pharmacare: information for Nova Scotians. Halifax (NS): Province of Nova Scotia, Department of Health and Wellness; [cited 2018 Oct 25]. Available from: https://novascotia.ca/dhw/pharmacare/nova-scotians.asp

6. Capital Health medication manual policy and procedure. Policy MM05-027: Funding of drugs and their administration in outpatient settings. Halifax (NS): Capital Health; 2014 Mar.

7. Chevalier B, Simpson C, Hemming H, Hiltz A, Monroe R, Sullivan V. Piloting an outpatient policy for funding drugs and their administration in rheumatology. Healthc Q. 2013;16(3):42-7.
8. Forte L, Olson C, Atchison C, Gesy, K. Reimbursement for supportive cancer medications through private insurance in Saskatchewan. Can J Hosp Pharm. 2009;62(3):232-7.

9. Kidney Disease: Improving Global Outcomes (KDIGO) Anemia Work Group. KDIGO clinical practice guideline for anemia in chronic kidney disease. Kidney Int Suppl. 2012;2(4):279-335.

10. Moist LM, Troyanov S, White CT, Wazny LD, Wilson J, McFarlane P, et al. Canadian Society of Nephrology commentary on the 2012 KDIGO clinical practice guidelines for anemia in CKD. Am J Kidney Dis. 2013; 62(5):860-73

Jo-Anne S Wilson, BSCPharm, ACPR, PharmD, is with the College of Pharmacy, Faculty of Health, Dalhousie University, and the Division of Nephrology, Department of Medicine, Nova Scotia Health Authority, Halifax, Nova Scotia.

Jaclyn Tran, BScPharm, ACPR, is with the Nova Scotia Renal Program, Nova Scotia Health Authority, Halifax, Nova Scotia.

Annette Veith, RPhT, is with the Nova Scotia Renal Program, Nova Scotia Health Authority, Halifax, Nova Scotia.

David Landry, BSc, MN, RN-NP, is with the Nova Scotia Renal Program, Nova Scotia Health Authority, Halifax, Nova Scotia.

Heather Neville, BScPharm, MSc, FCSHP, is with the Department of Pharmacy, Nova Scotia Health Authority, Halifax, Nova Scotia.

Cindy Kelly, RN, BScN, is with the Nova Scotia Renal Program, Nova Scotia Health Authority, Halifax, Nova Scotia.

Steven Soroka, MD, MSc, FRCPC, is with the Division of Nephrology, Department of Medicine, Nova Scotia Health Authority, and the Division of Nephrology, Faculty of Medicine, Dalhousie University, Halifax, Nova Scotia.

Kenneth West, MD, FRCPC, is with the Division of Nephrology, Department of Medicine, Nova Scotia Health Authority, Halifax, Nova Scotia.

Competing interests: None declared.

Address correspondence to:

Dr Jo-Anne S Wilson

Division of Nephrology, Department of Medicine

Dalhousie University

Room 5092, Dickson Building

5820 University Avenue

Halifax NS B3H 2 Y9

e-mail: jo-anne.wilson@dal.ca

Funding: None received.

Acknowledgements: The authors would like to acknowledge Maria Harlow-Gillighan, BScPharm candidate, for help with organization of the manuscript, and Stephanie Boudrea, RPhT, and Heather Hemming, BSW, for their involvement during the feasibility pilot study. 